\title{
The Scanning Laue Nanodiffraction Microscopy at Taiwan Photon Source
}

\author{
Ching-Shun $\mathrm{Ku}^{1,2, *}$, Shang-Jui Chiu ${ }^{1}$, Ching-Yu Chiang and Chia-Hsien Lin ${ }^{1}$
}

${ }^{1 .}$ Materials Science Group, Scientific Research Division, National Synchrotron Radiation Research Center (NSRRC), Hsinchu, Taiwan.

2. Graduate Program for Advanced Light Source, National Tsing-Hua University, Hsinchu, Taiwan.

*Ching-Shun Ku, csku@nsrrc.org.tw

The X-ray Nanodiffraction Beamline (XND) is one of the phase-I projects for Taiwan Photon Source (TPS) in NSRRC. The end-station called "FORMOSA" (FOcusing x-Ray for MicrO-Structural Analysis) is dedicated to the use of white/mono-beam Laue diffraction for structural analysis. For instance, users could obtain the 2D and 3D distribution of crystal phases, orientations, residual stain/stress, and dislocations for materials in a complex form without annoying specimen preparation and distorting the sample during measurement. The current spatial resolution is better than $80 \times 80 \mathrm{~nm}$ at lateral plane. Furthermore, FORMOSA also provides many complementary tools other than x-ray. The Tetra-probes could deploy several scanning probe methods such as atomic force microscopy, scanning tunneling microscopy and scanning near-field optical microscopy to collect surface, electrical, and optical properties of specimen together with structural information from Laue simultaneously; the projection x-ray detector for absorption contrast imaging; the x-ray fluorescence detector providing elemental information; the integrated hexapod scanner providing nanometer resolution in 6-degree of freedom to carry on user's own sample holder for precision measurement, and the cryogenic stage integrated with heater for temperature dependence experiments. Particularly, FORMOSA is equipped with high-resolution field emission scanning electron microscopy (FESEM) inside the end-station to obtain real-time imaging of specimen. This SEM sophisticated aligned with focusing $\mathrm{x}$-ray beam at the same position on the sample surface, enables users to find out the regions of interest on the screen and obtain the corresponding diffraction pattern instantly. All these equipment were meticulous arranged in a tiny space inside the analysis chamber of FORMOSA as shown in figure 1. In summary, XND beamline and FORMOSA end-station provide not only 2D/3D-Laue Microscopy but also nano-XRF, nano-XAS, nano-XEOL/CL, SPM and SEM information for diverse research programs.

In this presentation, we will briefly introduce to you the design and commissioning results for XND and FORMOSA. In addition, the presenter also will cover some very preliminary and exciting results from users research projects in the areas of ultra-thin films, emergent materials and nanostructures to demonstrate the capabilities of FORMOSA.

References:

[1] Xian Chen, Catherine Dejoie, Tengfei Jiang, Ching-Shun Ku and Nobumichi Tamura, MRS Bulletin 41, (2016), p. 445. 


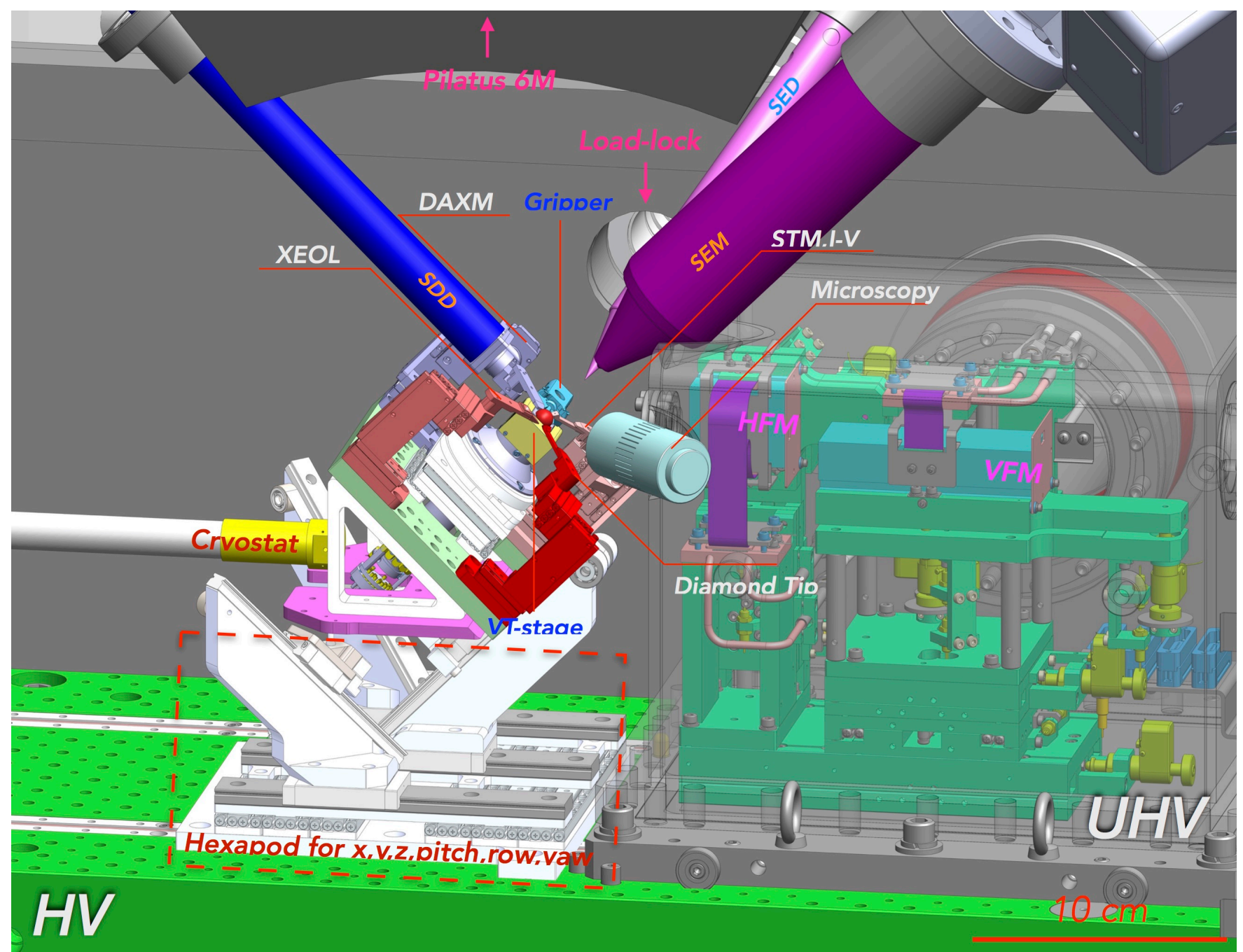

Figure. 1. An inside view of the future FORMOSA end-station at the Taiwan Photon Source in Taiwan. [1] 